[When citing this chapter, refer to Behaviour 152 (2015) 461-492]

\title{
A comparative assessment of handedness and its potential neuroanatomical correlates in chimpanzees (Pan troglodytes) and bonobos (Pan paniscus)
}

\author{
William D. Hopkins ${ }^{\mathrm{a}, \mathrm{b}, *}$, Jennifer Schaeffer ${ }^{\mathrm{b}}$, Jamie L. Russell $^{\mathrm{b}}$, \\ Stephanie L. Bogart ${ }^{\mathrm{a}}$, Adrien Meguerditchian ${ }^{\mathrm{c}}$ and Olivier Coulon ${ }^{\mathrm{d}}$ \\ ${ }^{a}$ Neuroscience Institute and Language Research Center, Georgia State University, \\ Atlanta, GA 30302, USA \\ ${ }^{\mathrm{b}}$ Division of Developmental and Cognitive Neuroscience, Yerkes National Primate \\ Research Center, 954 Gatewood Road, Atlanta, GA 30029, USA \\ ${ }^{c}$ Laboratory of Cognitive Psychology, UMR 7290, Aix-Marseille University, \\ CNRS, Marseille, France \\ d Aix-Marseille Universite, LSIS, UMR, CNRS 7296 Marseille, France \\ *Corresponding author's e-mail address: whopkins4@gsu.edu or whopkin@emory.edu
}

Accepted 31 March 2014; published online 8 May 2014

\begin{abstract}
The evolutionary origins of human right-handedness remain poorly understood. Some have hypothesized that tool use served as an important preadaptation for the eventual evolution of populationlevel right-handedness. In contrast, others have suggested that complex gestural and vocal communication served as prerequisite for the evolution of human right-handedness. In this study, we tested these competing hypotheses by comparing the handedness of bonobos and chimpanzees, two closely related species of Pan, on three different measures of hand use including simple reaching, manual gestures and coordinated bimanual actions. Chimpanzees are well known for their tool using abilities whereas bonobos rarely use tools in the wild. In contrast, many have suggested that bonobos have a more flexible gestural and vocal communication system than chimpanzees. The overall results showed that chimpanzees were significantly more right-handed than bonobos for all three measures suggesting that adaptations for tool use rather than communication may have led to the emergence of human right-handedness. We further show that species differences in handedness may be linked to variation in the size and asymmetry of the motor-hand area of the precentral gyrus. The results are discussed within the context of evolutionary theories of handedness, as well as some limitations in the approach to handedness measurement in nonhuman primates.
\end{abstract}

\section{Keywords}

handedness, bonobos, chimpanzees, central sulcus morphology, brain asymmetry, motor skill. 


\section{Introduction}

Chimpanzees (Pan troglodytes) and bonobos (Pan paniscus) are the closest evolutionary relatives to humans. Although humans split from the genus Pan 5-7 million years ago, bonobos and chimpanzees shared a common ancestor as recently as 1-2 million years ago (Fischer et al., 2004; Won \& Hey, 2005). The vast majority of cognitive and behavioral studies in captivity have been done with chimpanzees, with relatively little information on the bonobo, likely due to the greater availability of chimpanzees compared to bonobos. However, even with the relative scarcity of bonobo data, both species have shown similar cognitive abilities, including for example: symbolic acquisition (Brakke \& Savage-Rumbaugh, 1995, 1996; Lyn, 2007), symbol ordering (Greenfield \& Savage-Rumbaugh, 1990; Lyn et al., 2011), representational (pretend) play (Lyn et al., 2006) and mirror self-recognition (Gallup, 1970; Hyatt \& Hopkins, 1994). Although the physical resemblance and the recently recorded cognitive similarities are manifest, the two species have markedly different social structures and behavioural patterns (de Waal, 1989; Stanford, 1998).

For instance, studies in captivity and in the wild show that bonobo society is decidedly matriarchal, as opposed to the alpha-male dominated chimpanzee society (Parish, 1996; Parish et al., 2000). In addition there is a greater number of detailed reports of tool manufacture and use in chimpanzees (Whiten et al., 1999, 2001), leading some experts to declare bonobo culture as less 'material' (McGrew, 1992), albeit far fewer studies have been conducted in Pan paniscus (Toth et al., 1993; Gruber et al., 2010). In contrast, the reports of communication abilities suggest that while both species are highly communicative, bonobos may have a somewhat more flexible and sophisticated communicative system than chimpanzees (Hopkins \& Savage-Rumbaugh, 1991; Taglialatela et al., 2003; Pika et al., 2005; Pollick \& de Waal, 2007; Clay \& Zuberbuhler, 2011). More generally, it has been suggested that bonobos perform better than chimpanzees on tasks that assess different dimensions of social cognition or cooperation whereas chimpanzees perform better on competitive, physical and spatial cognition (Hare \& Tomasello, 2004; Hare, 2007; Hare et al., 2007; Herrmann et al., 2010; Rosati \& Hare, 2012).

The purpose of the current study was to examine handedness in chimpanzees and bonobos as a means of assessing potential differences in lateralization of the nervous system as it relates to motor functions. Our particular 
interest in this question stems from historical and contemporary theories on the evolution of right-handedness in humans in relation to emergence of higher cognitive and motor functions including language and speech, tool use and bipedalism (Corballis, 1992; Bradshaw \& Rogers, 1993; Bradshaw, 1997). For example, it has been postulated that human right-handedness is a consequence of selection for increasing motor demand for oro-facial motor control or gestural communication (Annett, 2002; Corballis, 2002). Because it has been reported that bonobos have greater flexibility in vocalizations and gestures than chimpanzees (Savage-Rumbaugh, 1984; Hopkins \& SavageRumbaugh, 1991; Taglialatela et al., 2003; Pollick \& de Waal, 2007), it could be hypothesized that they would express more pronounced asymmetries in manual gestures compared to chimpanzees. In contrast, others have suggested that the cognitive and motor demands of tool-use may have served as a prerequisite to the evolution of complex praxic functions that are primarily under control of the left hemisphere (Frost, 1980; Greenfield, 1991; Gibson \& Ingold, 1993; McGrew \& Marchant, 1997). As noted above, tool-use is widespread in chimpanzees compared to bonobos and there is evidence of population-level handedness in wild chimpanzees for some forms of tool-use such as leaf sponging, ant-dipping and termite fishing but not nut-cracking (Boesch, 1991; McGrew \& Marchant, 1992, 1996, 1997; Biro et al., 2003; von Lonsdorf \& Hopkins, 2005; Biro et al., 2006; Marchant \& McGrew, 2007; Humle \& Matsuzawa, 2009; Bogart et al., 2012b). If this theory is correct, then it might be hypothesized that chimpanzees would show greater expression of manual asymmetries for object directed actions compared to bonobos (see also Forrester et al., 2011, 2012). To test these competing hypotheses, we tested a sample of chimpanzees and bonobos on three measures of handedness including manual gestures, simple reaching, and a task measuring coordinated bimanual actions, referred to as the TUBE task.

Beyond the theoretical predictions for handedness in these two species, there are also behavioral and neurological reasons to predict potential differences in handedness. For instance, Hopkins et al. (2009) recently reported that bonobos showed greater leftward asymmetries in the motor-hand area of the precentral gyrus (or KNOB) as well as striatal structures including the putamen and caudate. In humans and chimpanzees, asymmetries in the KNOB region correlate with handedness with a larger KNOB volume found in the hemisphere contralateral to the subject's preferred hand (Hammond, 2002; Hopkins \& Cantalupo, 2004). Based on the report by Hopkins et al. 
(2009) of a larger left KNOB region in bonobos, it can be hypothesized that this species should show more pronounced right-handedness compared to the chimpanzees. With respect to their behaviour, several reports of handedness in bonobos also suggest potential species differences. Chapelain and colleagues (Chapelain \& Hogervorst, 2009; Chapelain et al., 2011) have examined handedness for the TUBE task, and contrary to chimpanzees (Hopkins et al., 2004; Llorente et al., 2010), they reported no evidence of populationlevel right handedness in the bonobos (see also Hopkins et al., 2011). There are also reports of a lack of population-level handedness for manual gestures in bonobos by several authors (Hopkins \& de Waal, 1995; Shafer, 1997; Harrison \& Nystrom, 2008), which also contradicts reports in captive chimpanzees (Hopkins et al., 2005a), though methods of handedness assessment may explain some of these differences (Hopkins et al., 2012). Whether these purported species differences reflect methodological factors or are true differences is not clear because the approaches to the assessment of handedness have varied between studies. Thus, the aim of this study was to compare bonobos and chimpanzees on identical measures of handedness when using the same procedures. In addition to hand preferences, we also measured grasping morphology and performance asymmetries when picking up small food items in the bonobos and chimpanzees. Christel et al. (1998) reported that bonobos show nearly exclusive grasping responses that involve the thumb and index finger, notably the lateral portion. In contrast, Hopkins and colleagues (Hopkins et al., 2002, 2005b) have reported that, though some chimpanzees prefer to use the thumb-index grasping response, many prefer to use the index and middle finger to grasp small food items, a pattern apparently rarely observed in the three bonobos studied by Christel et al. (1998). Morphologically, the hand of the bonobo and chimpanzees are quite similar; thus the reports of potential differences in grasping morphology may reflect underlying differences in neural innervations of the digits or other morphological features that may be involved with reach-and-grasp responses such as the wrist and elbow. To further explore whether species differences are evident in grasping morphology, we compared the bonobos' and chimpanzees' grasping responses when picking up small food items. We also assessed whether performance differences in grasping skill were evident between the chimpanzees and bonobos. Previous studies in chimpanzees have shown that they exhibit a small but significant right hand advantage in grasping skill (Hopkins \& Russell, 2004) but, to our knowledge, there are no such 
data available in bonobos. Christel et al. (1998) assessed response times during reach-and grasp responses in three bonobos and found that latencies to reach were shorter for the right compared to left hand but that the time needed to grasp the objects was slightly faster for the left compared to right hand. Comparing the results by Christel et al. (1998) with the findings by Hopkins \& Russell (2004) is difficult because different dimensions of motor skill were measured. Thus, in this study, we assessed both chimpanzees and bonobos on the same grasping task in order to compare lateralization in motor skill.

Finally, we present here some preliminary findings on comparative differences in cortical organization between chimpanzees and bonobos. Despite the intense interest in behavioural and cognitive abilities between chimpanzees and bonobos, there is remarkably little research on how these potential differences might map onto variation in cortical organization. Hopkins et al. (2009) measured the volume and areas of eight brains regions in a sample of seven chimpanzees and bonobos and found that bonobos showed greater leftward asymmetries for the motor-hand area of the precentral gyrus, putamen, caudate and hippocampus. More recently, Rilling et al. (2012) used both voxel-based morphometry (VBM) and diffusion tensor imaging (DTI) to compare cortical connectivity in three chimpanzee and bonobo brains. These authors found that bonobos had more grey matter than chimpanzees in the anterior amygdala and insular cortex compared to chimpanzees. Rilling et al. also found significantly larger cortical pathways connecting the amygdala and anterior cingulate cortex compared to the chimpanzees.

Rather than use region-of-interest or VBM analysis, here we examined species difference in the surface area and cortical folding of the central sulcus (CS) using a software program called BrainVisa. BrainVISA 4.1.1 (BV) is freely distributed software (available online at http://brainvisa.info) that measures cortical folding of the brain (Mangin et al., 2004). We selected the CS because the cortex lying anterior and posterior to the CS are the primary motor and somatonsensory cortex, the cortical region where all motor and sensory regions are represented in the brain, most notably the hand (Bailey et al., 1950). For instance, stimulation of the central region of the CS will result in selective movements of the hand and individual digits, suggesting that this is the cortical representation of the hand. Furthermore, there is an anatomical landmark within the precentral gyrus, referred to as the KNOB (Yousry et al., 1997), and several studies have shown that the volume 
and shape of the KNOB differs in right- and left-handed individuals (Boling et al., 1999; Hammond, 2002) and similar results have been reported in chimpanzees and capuchin monkeys (Hopkins \& Cantalupo, 2004; Phillips \& Sherwood, 2005; Dadda et al., 2006). The appearance of the KNOB in the brain has evolved as a consequence of the CS having to fold over and around a buried gyrus, referred to as the pli-de-passage frontoparietal moyen parietale (PPFM), which connects the precentral and postcentral gyrus, and was originally described by Broca (Alkadhi \& Kollias, 2004). Comparative studies have shown that the KNOB is only evident in great apes and humans and that this is likely attributable to increased gyrification of the brain due to increasing size and connectivity (Hopkins \& Pilcher, 2001). Further, using different parameterization tools within $\mathrm{BV}$, the depth of the CS can be quantified along the entire superior to inferior plane (Coulon et al., 2006; Cykowski et al., 2008; Hopkins et al., 2010). This procedure allows for a means of quantifying the PPFM within the entire surface area of the CS. In this study, we examined the surface area and folding pattern of the entire CS as well as the region within the CS corresponding to the PPFM in a sample of chimpanzees and bonobos to test whether (a) differences are evident in CS morphology and (b) whether these differences might map onto variation in motor skill and/or handedness.

\section{Methods}

\subsection{Subjects}

At least one measure of handedness was available in 34 bonobos and 34 chimpanzees that were matched on age, sex and rearing histories (see Table 1). The bonobos were housed at three facilities including the Jacksonville Zoo and Gardens, Milwaukee County Zoo and Great Ape Trust of Iowa. The chimpanzees were all housed at the Yerkes National Primate Research Center of Emory University. Within each species, there were 19 females and 15 males and the apes ranged from 3 to 44 years of age (mean $=20.34$ years, $\mathrm{SE}=1.96$ ). Within each cohort, there were three wild-caught and 31 captive-born individuals. Within the captive-born cohort, there were 27 mother-reared and 4 human-reared individuals. Human-reared apes were those that were removed from their natal groups before 1 month of age and raised in a human nursery setting until they were old enough to be introduced into conspecific social groups. Mother-reared apes were those that were born and raised by their conspecific mothers and raised in nuclear family settings. 


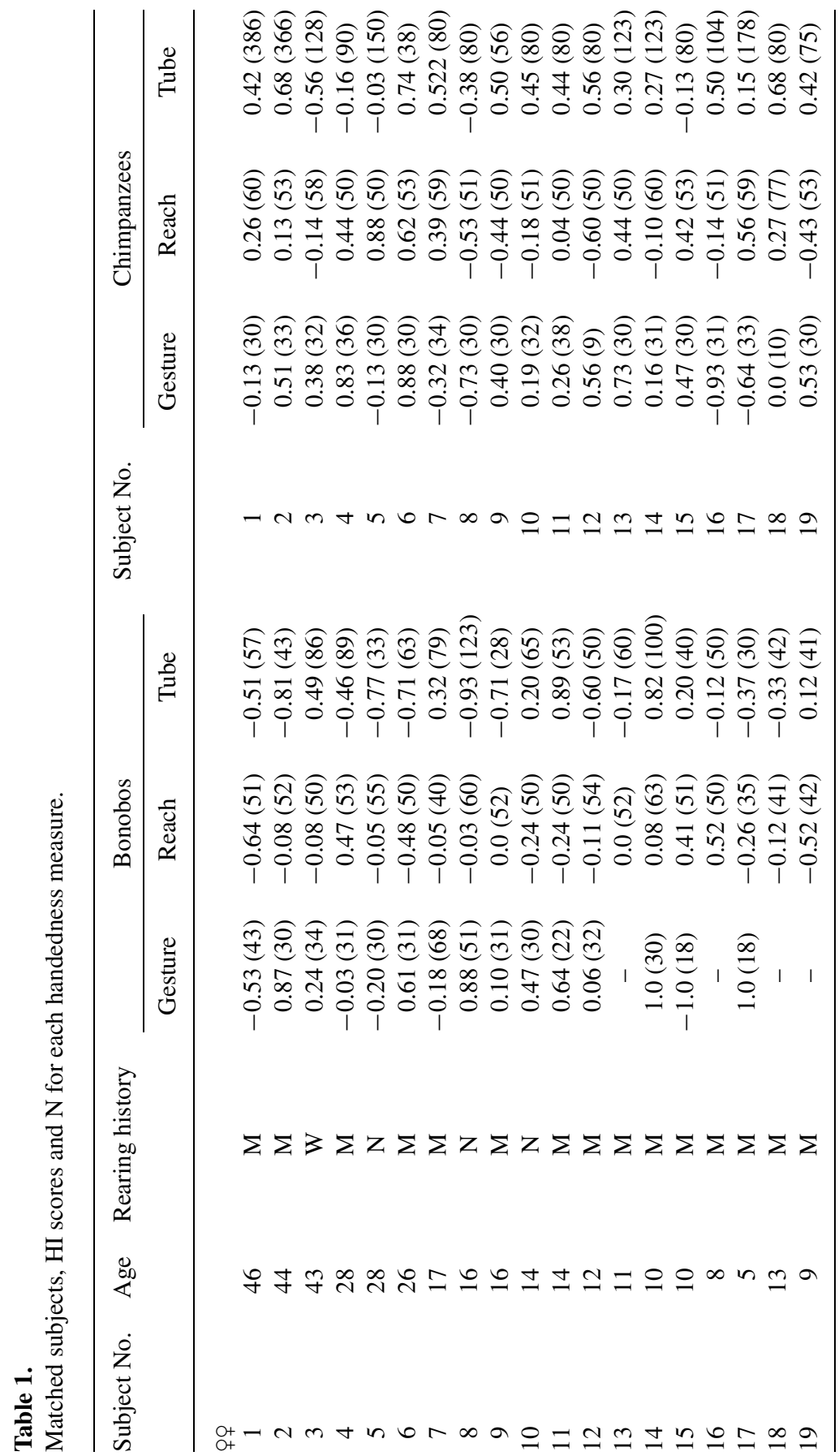




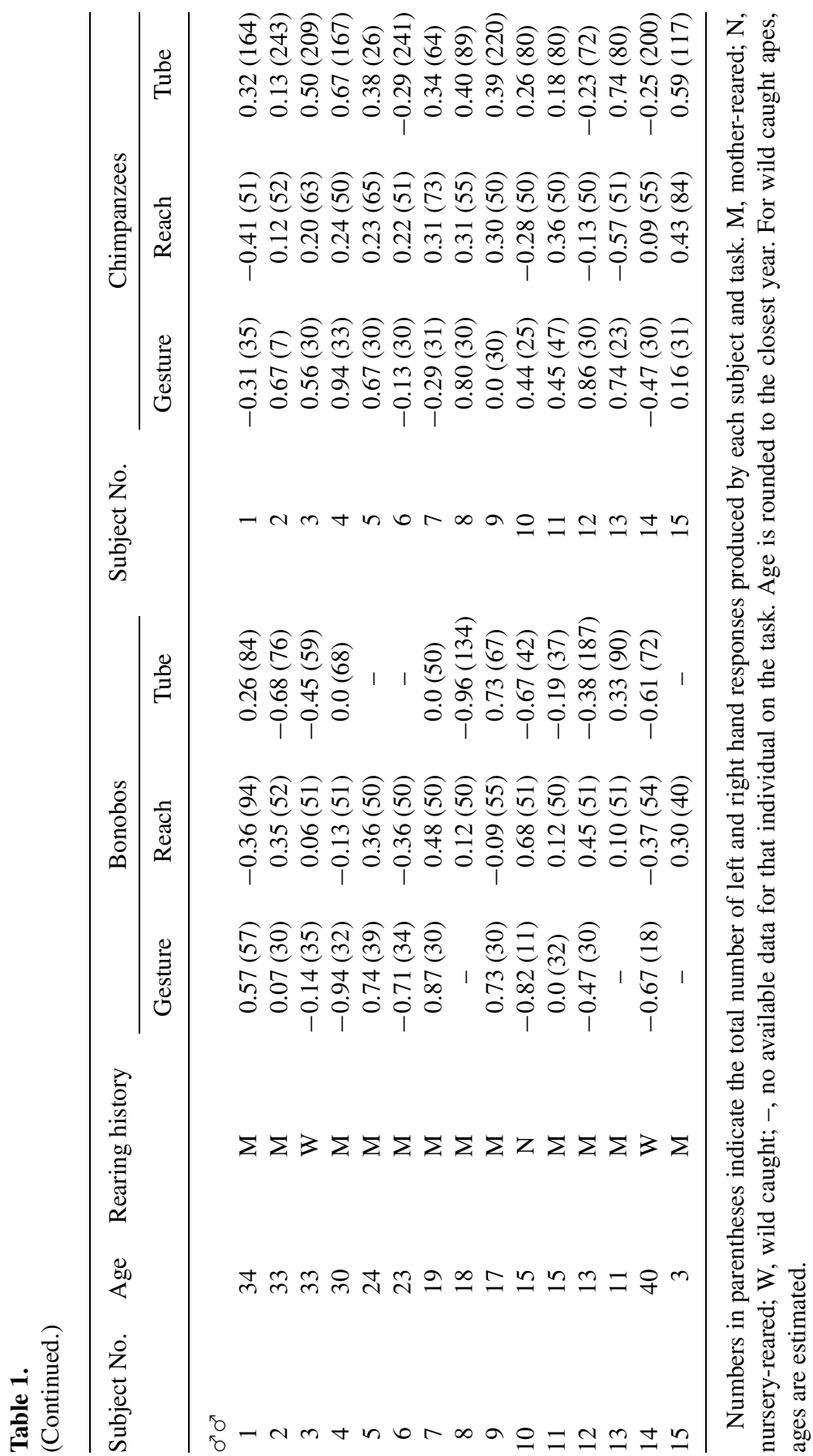




\subsection{Procedure}

Handedness was assessed on three measures of hand use previously described in these subjects including manual gestures, simple reaching, and a task measuring coordinated bimanual actions, referred to as the TUBE task (Hopkins, 1995a; Hopkins et al., 2005a, b, 2007). These three measures were selected because they elicited different dimensions of hand preferences and have been shown to be reliable measures across time (Hopkins, 2007). A brief description of each measure is provided below. The testing was done in the indoor and outdoor enclosures of the apes by three different experimenters. The three experimenters participated in data collection from all individuals between the two species. Thus, no one experimenter solely collected data from a single species. Because hand use and grasping morphology were fairly overt manual actions, we did not calculate interrater reliability in their assessment between raters prior to the onset of data collection.

\subsubsection{Manual communicative gestures}

At the onset of each trial, an experimenter would approach the subject's home cage and centre themselves in front of the subject at a distance of approx. 1.0-1.5 m. If the subject was not already positioned in front of the experimenter at the onset of the trial, the subject would immediately move towards the front of the cage when the experimenter arrived with the food. The experimenter then spoke the ape's name and offered a piece of food until the subject produced a manual gesture, whereupon they were given the food. Only responses in which the apes' unimanually extended the digit(s) through the cage mesh to request the food were considered a response. Hand use was recorded as right or left for each response. Other possible manual responses such as cage banging or clapping were not counted as a gesture. Two-handed gestures, although rare, were not scored as were gestures that were produced by the subject prior to the experimenter arriving in front of the subject's home cage. A minimum of 30 responses were collected from most subjects (range 7-57) (Hopkins et al., 2005c) and the number of trials administered on a given day varied with the subject's motivation and availability for testing. However, at least 5 min separated the administration of each trial. Subjects were tested in both the indoor and outdoor sections of their home enclosures. 


\subsubsection{Coordinated bimanual task (TUBE)}

The second handedness measure was a task requiring bimanual coordinated actions, referred to as the TUBE task (Hopkins, 1995a). For the TUBE task, peanut butter or other foods that had adhesive qualities were smeared on the inside edges of poly-vinyl-chloride (PVC) tubes approx. $15 \mathrm{~cm}$ in length and $2.5 \mathrm{~cm}$ in diameter. Peanut butter was smeared on both ends of the PVC pipe and was placed far enough down the tube such that the subjects could not lick the contents completely off with their mouths but rather had to use one hand to hold the tube and the other hand to remove the substrate. The PVC tubes were handed to the subjects in their home cages and a focal sampling technique was used to collect individual data from each subject. The hand of the finger used to extract the peanut butter was recorded as either right or left by the experimenter. Each time the subject reached into the tube with their finger, extracted peanut butter and brought it to their mouth, the hand used was recorded as left or right. Each subject was tested on at least two occasions and a minimum of 30 responses was obtained from each subject (range 30-386).

\subsubsection{Simple reaching}

During each trial, a raisin was thrown into the subject's home cage. The raisin was thrown by the experimenter to a location at least three meters from the focal subject so that the apes had to locomote to the raisin, pick up the raisin and bring it to their mouth for consumption. When the subject acquired the raisin, the experimenter recorded the hand used as left or right. One, and only one reaching response, was recorded each trial to assure independence of data points. Thus, raisins were not randomly scattered in home cages but rather an individual raisin was thrown into the cage and the subject retrieved the raisin before another was thrown into the cage. Subjects were required to locomote at least three strides between reaching responses to maintain postural readjustment between trials. A minimum of 35 responses was collected from each subject with the range between 35 and 94 for the sample.

In addition to hand use during the simple reaching task, we also recorded the type of grip used by the apes when grasping the raisins. Grip type was characterized as either thumb-index, middle-index, or single digit. Thumbindex gripping was recorded when the subjects abducted the thumb to the lateral or tip of the index finger to grip the food item. Gripping was recorded 
as middle-index grip when the subjects grasped the food between the index and middle finger with the hand either in a prone or supine position. Thumb-index and middle-index grip types were by far the most common, but occasionally subjects engaged in what was recorded as single digit responses. Single digit responses were instances in which the chimpanzees used one finger to press down hard enough on the food item so that it stuck while being taken to the mouth.

\subsection{Grasping errors}

For this measure, small food items (quartered peanuts, sunflower seeds, redhots or skittles) were thrown into the subject's home cage. The use of different food was based on the individual apes' preferences for these items as well as food restrictions at each facility (i.e. one bonobo from JZG was allergic to peanuts, so no peanuts are allowed in the Great Ape area). Food items were dropped in the cage until exactly twenty successful grasping responses with each hand had been obtained from each subject. Because the emphasis of this particular experiment was on obtaining twenty responses with each hand from each subject, the subjects were not required to locomote to the food items in order for the response to be recorded. Instead, the food items were dropped next to one of the subject's hands, thus encouraging them to use one hand over the other. The experimenter watched as the subject attempted to pick up the food item and noted any errors made in grasping the food. An error was recorded when the subjects attempted to grasp but dropped the food or failed to grasp the food item from the floor upon their attempt. If at any point the subject switched hands or used their mouth to obtain the food, the trial was not included. Data were collected ad libitum and during testing, the foods were thrown to either side of the subject's body in a random order in order to facilitate responses with both hands.

\subsection{Grasping data analysis}

For each hand preference and performance measure, a handedness index (HI) was derived following the formula $(\mathrm{HI}=(R-L) /(R+L))$ where $R$ and $L$ represent the frequency of right and left hand use for each task, respectively. For the hand preference measures, positive HI values indicated a rightward asymmetry and negative values indicated a leftward asymmetry. Note that for the performance task, a positive HI would reflect better performance by the left compared to right hand, reflecting the fact that they would thereby make 
more errors with the right hand. For each handedness task, binomial $z$-scores were calculated for each subject based on the total frequency of left and right hand use. Subjects with $z$-scores greater than or equal to 1.96 or less than or equal to -1.96 were classified as right-handed and left-handed respectively while all other subjects were classified as ambiguously-handed $(z>-1.96$ and $z<1.96)$. Finally, for the grasping morphology analyses, we computed a percentage of thumb-index responses for each subject and hand by summing the total number of left and right thumb-index grasping responses and dividing these values by the total number of grasping responses for the left and right hands, then multiplying by 100 . Percentages varied between 0 and 100 with higher values indicating greater thumb-index grasping responses.

\subsection{Magnetic resonance image collection}

Magnetic resonance images (MRI) were obtained from 13 bonobos and 13 chimpanzees that were matched on age, sex and magnet strength. Four bonobos and 4 chimpanzees were scanned in vivo and the remaining 9 chimpanzees and 9 bonobos were scanned post-mortem. To minimize stress on the apes, all in vivo bonobo and chimpanzee magnetic resonance images were obtained during one of their annual physical examinations. Magnetic resonance image (MRI) scans followed standard procedures at the YNPRC. Thus, the animals were first sedated with ketamine $(10 \mathrm{mg} / \mathrm{kg}$ ) or telazol (3$5 \mathrm{mg} / \mathrm{kg}$ ) and were subsequently anaesthetized with propofol $(40-60 \mathrm{mg} / \mathrm{kg}$ per h). They were then transported to the MRI scanning facility and placed in a supine position in the scanner with their head in a human-head coil. Upon completion of the MRI, the apes were singly-housed for 2-24 h to permit close monitoring and safe recovery from the anesthesia prior to return to the home social group. All procedures were approved by the Institutional Animal Care and Use Committees at YNPRC and also followed the guidelines of the Institute of Medicine on the use of chimpanzees in research. The in vivo scans were obtained using a 1.5T G.E. echo-speed Horizon LX MR scanner (GE Medical Systems, Milwaukee, WI, USA). T1-weighted images were collected in the transverse plane using a gradient echo protocol (pulse repetition $=19.0 \mathrm{~ms}$, echo time $=8.5 \mathrm{~ms}$, number of signals averaged $=8$, matrix size $=256 \times 256$, with $0.7 \times 0.7 \times 1.2$ resolution). For the post-mortem scanning, either 4.7 or $7 \mathrm{~T}$ magnets were used and $\mathrm{T} 2$-weighted images were collected in the transverse plane using a gradient echo protocol (pulse repetition $=22.0 \mathrm{~s}$, echo time $=78.0 \mathrm{~ms}$, number of signals averaged $=8-12$, and a $256 \times 192$ matrix reconstructed to $256 \times 256$ ). 


\subsection{MRI processing}

Initially, using Analyze 8.1, all chimpanzee scans were skull-stripped, cropped, and reformatted at 0.7 cubic isotropic resolution and subsequently imported into BV. Extracting the sulci from the cortex in the brain scans involves a series of steps in a pipeline process within BV (Mangin et al., 2004) (see Figure 1) and these methods are described in detail elsewhere (Bogart et al., 2012a). Initially, the anterior and posterior commissures were manually specified on the MRI where they intersect with the mid-sagittal slice to align the brain. The first step was to correct for special inhomogeneities in the signal intensity providing a spatially smooth bias field with a stable distribution of tissue intensities (Figure 1b). Next, the analysis of the signal histogram

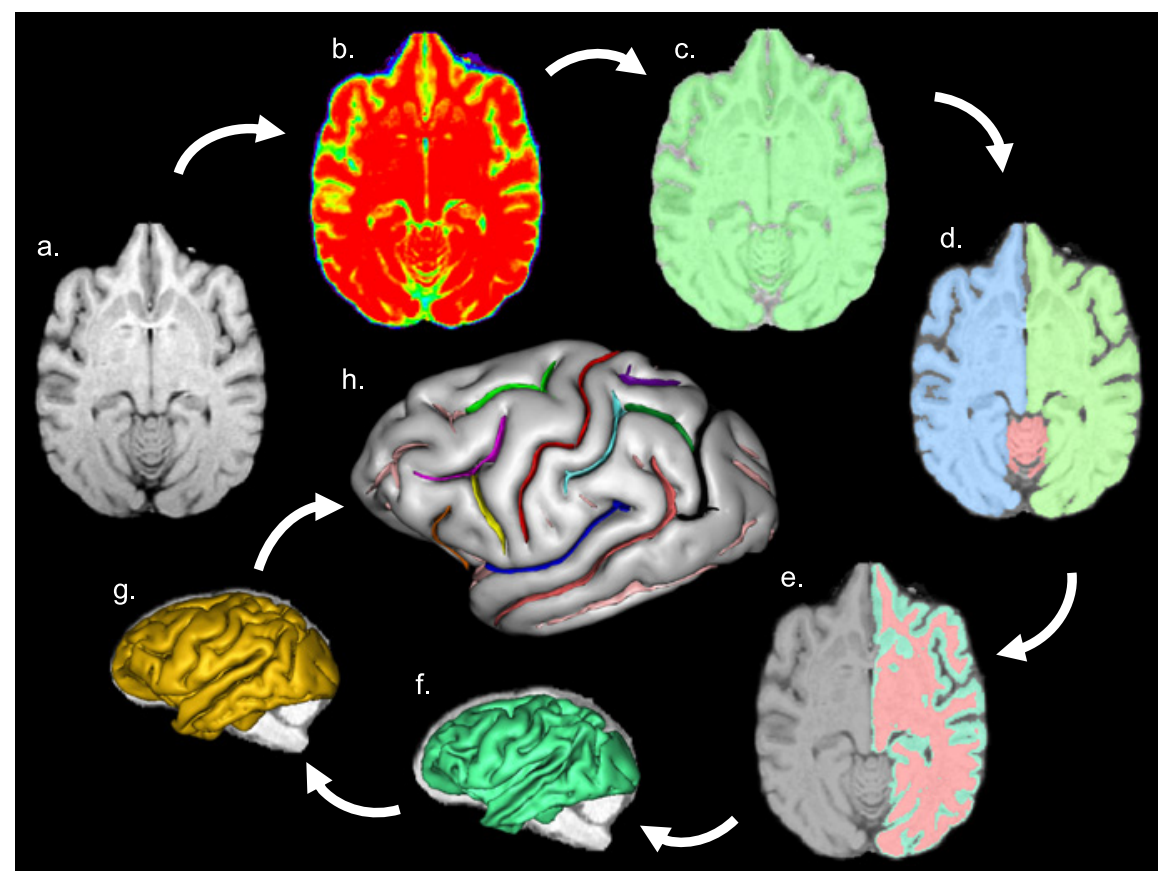

Figure 1. BrainVISA's pipeline processing steps. (a) MR image of a skull-stripped chimpanzee brain, (b) stable tissue intensities after bias field correction, (c) binary mask of the brain, (d) split mask of left and right hemispheres and cerebellum, (e) grey and white interface, (f) negative mould of the white matter, (g) skeletonised mould of cortical folding and (h) cortical fold graph of chimpanzee sulci with the central sulcus in red. This figure is published in colour in the online edition of this journal, which can be accessed via http:// booksandjournals.brillonline.com/content/journals/1568539x. 


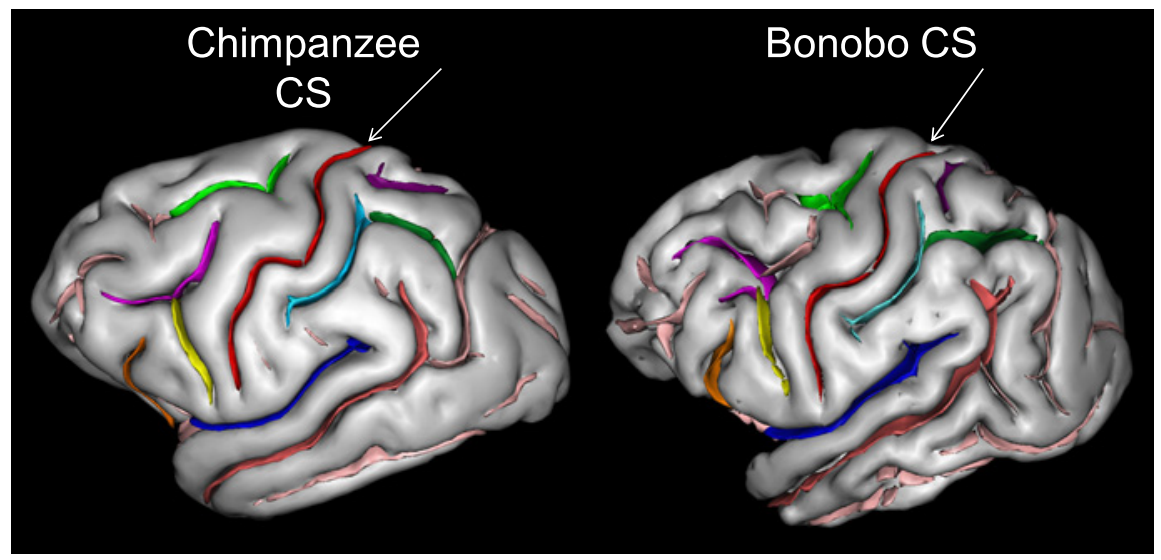

Figure 2. 3-D reconstruction of cortical sulci of the chimpanzee and bonobo brain with the central sulcus (red) indicated in each species. This figure is published in colour in the online edition of this journal, which can be accessed via http://booksandjournals.brillonline.com/ content/journals/1568539x.

and mathematical morphology was computed to obtain a binary mask of the brain (Figure 1c). The mask was then split into the left and right hemispheres and the cerebellum (Figure 1d). A negative mould of the white matter was computed from the split-brain mask. The outside boundary of this mould results from a $5 \mathrm{~mm}$ morphological closing of the masked hemisphere, filling up the folds. The grey/white interface is the inside boundary of the mould, reconstructed with topography, preserving deformations assuring the spherical topology of the mould (Figure 1e). Finally the mould was skeletonised to detect cortical folding, while topological constraints guarantee the resulting surfaces have no holes (Figure 1e, f). The deepest part of the fold indicates the buried gyrus given the grey/white interface. The final step results in creating a cortical fold graph containing all the extracted sulci (Figure 1h). The central sulcus was manually labelled on the 3-D renderings for each brain (Figure 2).

\subsection{Central sulcus measures}

Central sulcus measures included surface area $\left(\mathrm{mm}^{2}\right)$, mean depth $(\mathrm{mm})$ and grey matter thickness $(\mathrm{mm})$ (see Figure 3 ). The sulcus surface area was computed as the sum of the areas of all the triangles required to mesh the sulcus medial surface. The sulcus mean depth is the average depth computed across all the bottom points of the sulcus along its principal axis of projection 


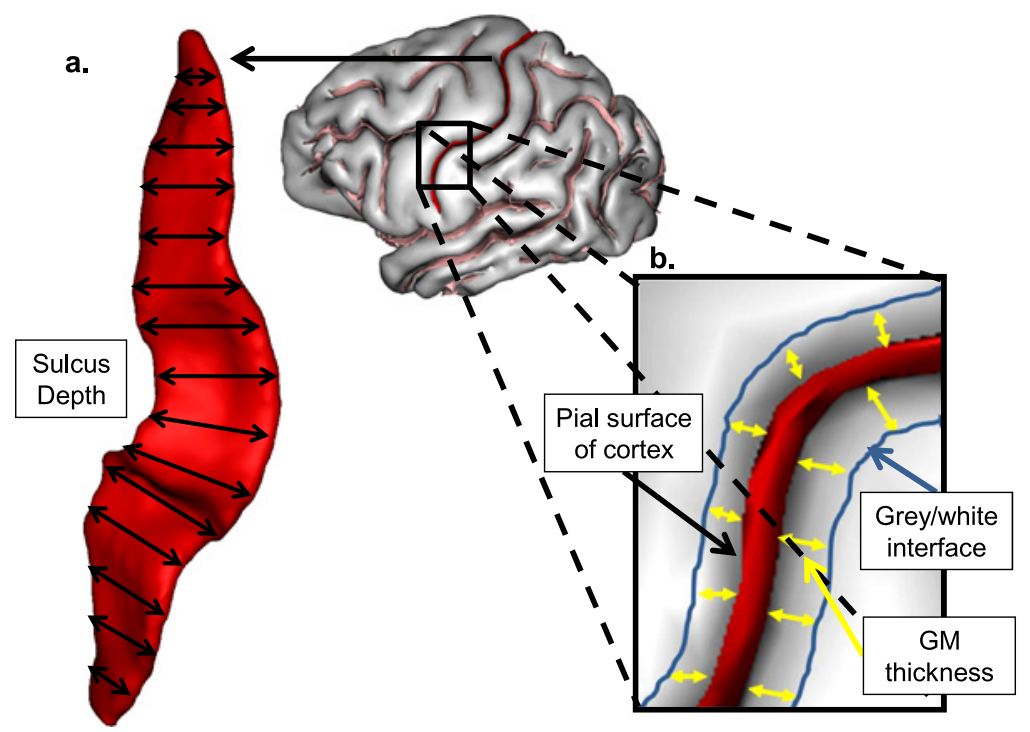

Figure 3. Chimpanzee central sulcus labeled and extracted. (a) The surface area and depth dimensions are shown in the extracted sulcus, as well as the $x$ - and $y$-coordinates used for computing differences in cortical folding of the CS along the dorsal-ventral axis. (b) Illustration of the landmarks used to quantify grey matter thickness. This figure is published in colour in the online edition of this journal, which can be accessed via http://booksandjournals. brillonline.com/content/journals/1568539x.

(i.e., dorsal-ventral or anterior-posterior). The bottom points are defined from topological properties and correspond to the sulcus edge that is not connected to the cortex hull (see Figure 3a). Hence, this definition can be used even with interrupted sulci. The depth of a bottom point is the length of the shortest path from this point to the cortex hull embedded in the sulcus medial surface (mathematically speaking, the shortest geodesic path). Sulcus grey matter thickness (GM thickness) was quantified by measuring the mean distance between the pial surface and the grey/white interface around the buried sulcus (see Figure 3b).

\subsection{Comparative analysis of the motor-hand area}

To explore the variation in the central CS region further, we quantified the CS surface area corresponding to the PPFM using previously described methods (Coulon et al., 2006; Cykowski et al., 2008; Hopkins et al., 2010) (see Figure 4). 


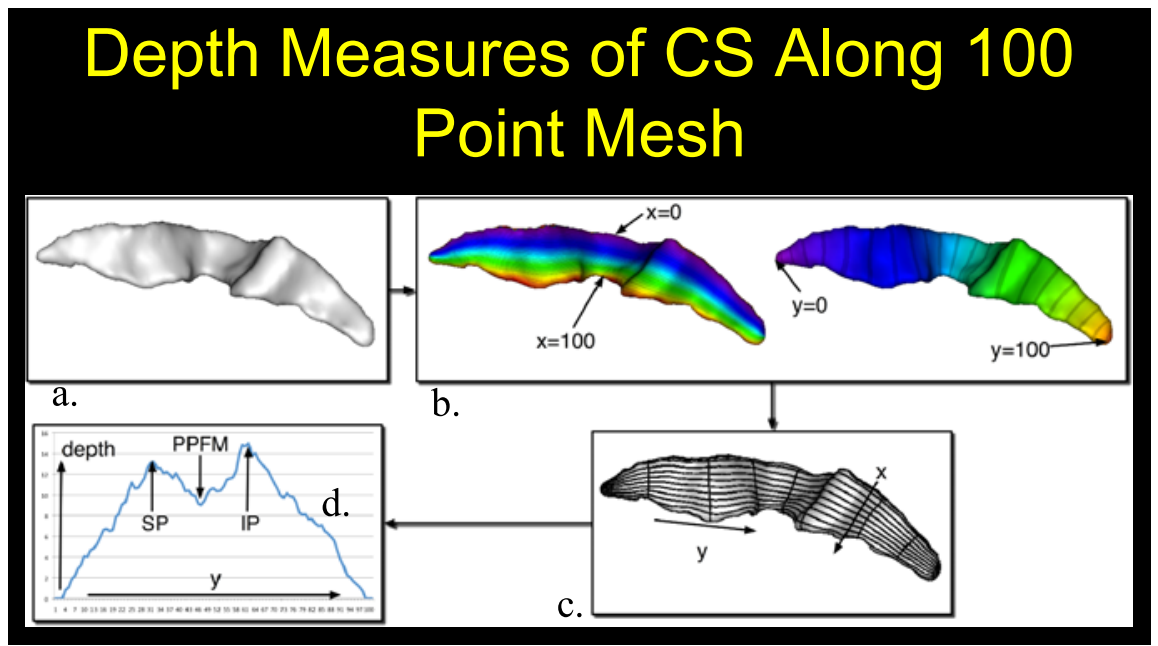

Figure 4. (a) Chimpanzee central sulcus and (b, c) the surface area and depth dimensions are shown in the extracted sulcus, as well as the $x$ - and $y$-coordinates used for computing differences in cortical folding of the CS along the dorsal-ventral axis (d) outputted data from CS parameterization. Depth of CS is plotted on ordinate and the $y$ coordinate along the abscissa. SP, superior maximum; CS, depth before $y$ coordinate 50; IP, maximum inferior depth after $y$ coordinate 50; PPFM, pli-de-passage moyen parietale, which is the shallowest CS depth measure between the SP and IP $y$ coordinates. This figure is published in colour in the online edition of this journal, which can be accessed via http://booksandjournals. brillonline.com/content/journals/1568539x.

For each subject, the CS was standardized into 100 equally spaced sections along the dorsal-ventral axis and the depth of the sulcus at each point was quantified. The procedure can be briefly summarized as follows: a coordinate system is computed on the sulcus that indicates the position of each point relative to its depth ( $x$ coordinate) and its position along the sulcus between the dorsal and ventral extremities ( $y$ coordinate), as illustrated in Figure 4b and 4c. At each position, $y=0$ through 100 along the sulcus length, the depth is defined as the length of the corresponding $y$ isocoordinate line (see Figures $4 \mathrm{~d}$ and 5). Various publications provide more technical details about this procedure (Davatzikos \& Bryan, 2002; Coulon et al., 2006; Cykowski et al., 2008; Hopkins et al., 2010). From the parameterization, the depth measure of the CS at each of the $100 y$-location points are outputted to a text file. From this file, we recorded the greatest depth measure that was superior (SP) and inferior (IP) to the central location of the CS (location 50) (see Figure 5). We then recorded the shallowest CS depth 


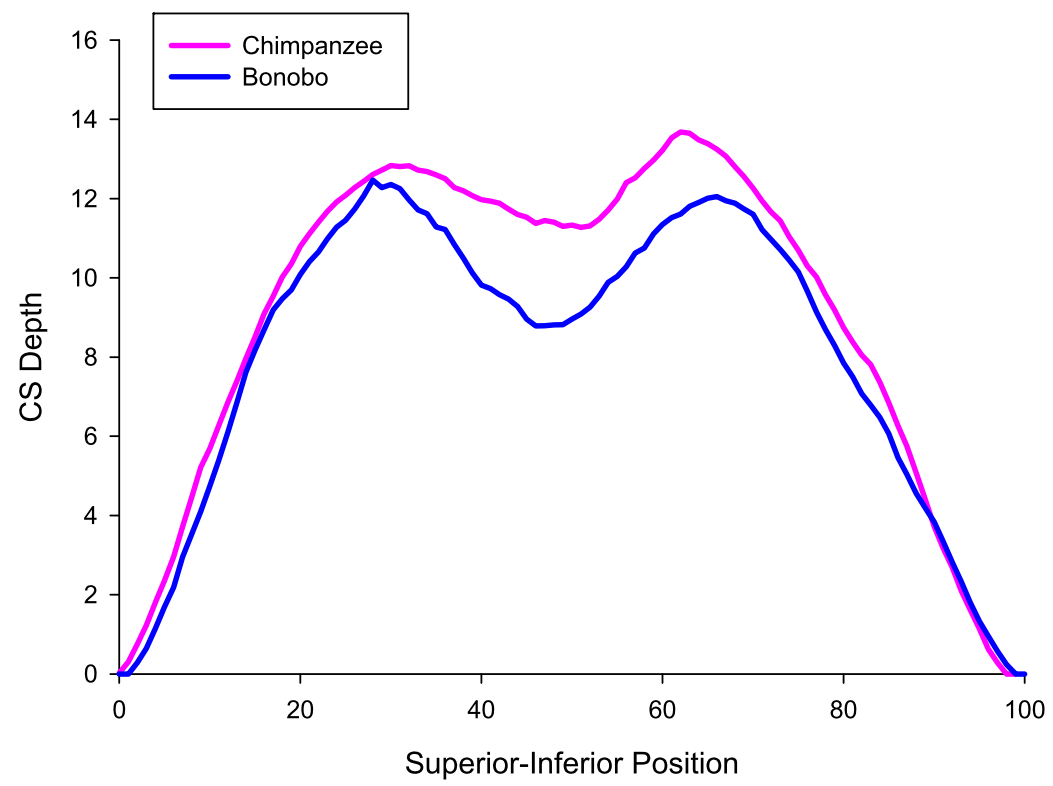

Figure 5. Variation in CS depth along the Superior-Inferior plane in the chimpanzee (purple line) and bonobos (blue line). SP, maximum superior depth; PPFM, shallowest CS depth point between SP and IP; IP, maximum inferior depth. This figure is published in colour in the online edition of this journal, which can be accessed via http://booksandjournals.brillonline. com/content/journals/1568539x.

between the SP and IP locations. The PPFM size was then computed for the left and right hemisphere following the formula $\left(\mathrm{PPFM}=\left(\left(\mathrm{depth}_{\mathrm{IP}}+\right.\right.\right.$ $\left.\left.\operatorname{depth}_{\mathrm{SP}}\right) / 2.0\right)-$ depth$\left._{\text {PPFM}}\right)$.

\subsection{CS data analysis}

For all measures (total CS surface area, mean depth, GM thickness), mean scores were computed by averaging the raw values between the left and right hemisphere. Asymmetry quotients (AQ) for all measures were also calculated following the formula: $(\mathrm{AQ}=(R-L) /(R+L))$, where $R$ and $L$ represent right and left hemisphere values for each measure, respectively. Positive AQ values reflected a right hemisphere bias whereas negative values reflected a left hemisphere bias. Inferential statistics were used for all analyses with alpha set to $p<0.05$ unless normality or homogeneity of variance assumptions for the tests could not be met. If these assumptions were not met, then non-parametric tests were used. Post-hoc comparisons on inferen- 
tial statistics, when used, were conducted using Tukey's Honestly Significant Difference test.

\section{Results}

\subsection{Hand preferences}

Individual HI scores for each subject are shown in Table 1 . We initially compared the HI values for each task as a function of the species and sex using a mixed model analysis of variance. Handedness measure was the repeated measure while species and sex were the between-group factors. A significant main effect for species was found $F_{1,56}=8.166, p<0.009$. Overall, the chimpanzees had significantly higher $\mathrm{HI}$ values than the bonobos and this was consistent across all three measures. The mean HI score for each species and task are shown in Figure 6. Within each species, we also performed one-sample $t$-tests to test for population-level asymmetries when considering handedness on a continuous scale of measurement. For all analyses, the estimated population mean for the HI scores was zero and none of the $t$-tests

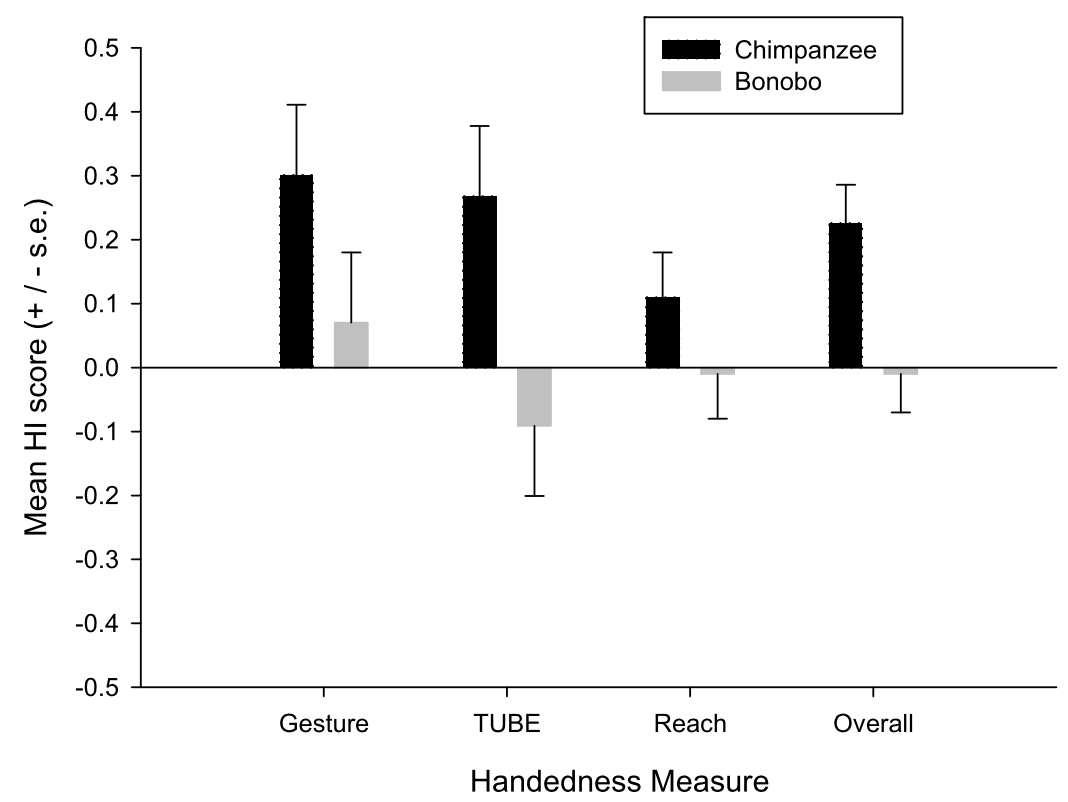

Figure 6. Mean HI scores ( \pm SE) for each handedness task (gesture, TUBE, and reach) and the overall score in bonobos and chimpanzees. 
Table 2.

Distribution of handedness based on $z$-scores for each task and species.

\begin{tabular}{lccccccc}
\hline \multirow{2}{*}{ Task } & \multicolumn{3}{c}{ Bonobo } & & \multicolumn{3}{c}{ Chimpanzee } \\
\cline { 2 - 4 } & No. R & No. A & No. L & & No. R & No. A & No. L \\
\hline Gestures & 12 & 7 & 9 & & 21 & 6 & 7 \\
TUBE & 16 & 4 & 11 & & 23 & 5 & 6 \\
Reaching & 9 & 15 & 10 & & 17 & 9 & 8 \\
\hline
\end{tabular}

reached statistical significance within the bonobo sample. In contrast, the chimpanzees showed population-level right handedness for manual gestures $t_{33}=3.27, p<0.01$ and the TUBE task $t_{33}=4.78, p<0.002$.

The hand preference data based on the classification criteria applied to the $z$-scores largely confirmed analysis of the HI scores. The hand preference distributions based on the $z$-scores are shown in Table 2. Within the bonobo sample, chi-square goodness-of-fit tests failed to reveal significant differences in the number of right- and left-handed individuals for any of the measures. In contrast, for chimpanzees, there were significantly more rightthan left-handed individuals for manual gestures $\chi^{2}(1, N=28)=7.00$, $p<0.02$ and the TUBE task $\chi^{2}(1, N=29)=9.97, p<0.004$.

\subsection{Grip morphology and performance asymmetries}

We next considered whether species and/or sex differences were evident in the grasping morphology and performance asymmetries. We initially calculated the percentage of grasping responses that involved the thumb and index finger for the left and right hands and compared these values between species and sexes using a mixed model analysis of variance. Hand was the repeated measure (left, right) while sex and species served as the between group factors. No significant main effects or interactions were found. The mean percentage of thumb-index grasping responses for bonobos and chimpanzees were 71.6 and $62.9 \%$, respectively. For the grasping error data, overall the chimpanzees (mean $\mathrm{HI}=-0.078$ ) showed a greater right hand advantage compared to the bonobos (mean $\mathrm{HI}=+0.013$ ) but the difference in HI values did not reach conventional levels of statistical significance.

\subsection{Central sulcus morphology and asymmetry}

In the first analysis, we compared the bonobos and chimpanzees on the mean surface area, mean depth and mean GM thickness of the overall CS using 
Table 3.

Descriptive data on CS and PPFM measurements in chimpanzees and bonobos.

\begin{tabular}{lcccc}
\hline & Bonobo & SD & Chimpanzee & SD \\
\hline Average & & & & \\
Surface area & 1063.08 & 193.85 & 1176.44 & 229.65 \\
Mean depth & 9.367 & 0.87 & 9.798 & 1.23 \\
GM thickness & 2.458 & 0.692 & 1.984 & 0.421 \\
PPFM & 6.227 & 2.629 & 5.167 & 1.995 \\
Asymmetry & & & & \\
Surface area & 0.042 & 0.123 & -0.048 & 0.171 \\
Mean depth & 0.029 & 0.054 & -0.046 & 0.111 \\
GM thickness & -0.042 & 0.092 & 0.008 & 0.038 \\
PPFM & -0.201 & 0.730 & 0.127 & 0.641 \\
Handedness & -0.010 & 0.331 & 0.215 & 0.291 \\
\hline
\end{tabular}

Surface area measurements are in $\mathrm{mm}^{2}$, while mean depth, GM thickness and PPFM measures are in $\mathrm{mm}$.

MANOVA. Species was the between group factors. A significant species difference was found for mean GM thickness $F_{1,24}=4.463, p<0.05$ with chimpanzees having thinner grey matter $($ mean $=1.984 \mathrm{~mm}$ ) compared to bonobos (mean $=2.459 \mathrm{~mm}$ ) (see Table 3 ). A second MANOVA using the surface area, mean depth and GM thickness AQ scores revealed significant species differences for the mean depth AQ $F_{1,24}=4.726, p<0.05$, while a borderline significant difference was found for GM thickness AQ variable $F_{1,24}=3.199, p<0.09$. The mean AQ scores for each species are shown in Table 3. For comparison, we have also plotted the mean HI scores. As can be seen, bonobos had greater leftward biases than chimpanzees for surface area and mean depth, but greater rightward biases for GM thickness. We next compared bonobos and chimpanzees on the PPFM measures within the CS region. A comparison in the mean PPFM, as well as the PPFM AQ values did not differ between the species; though there was a trend for bonobos to have a greater leftward asymmetry compared to the chimpanzees (see Table 3 ).

\section{Discussion}

The results of this study are relatively straightforward. When compared on three measures of hand preference, chimpanzees were significantly more right-handed than the bonobos. No species differences were found in performance asymmetries either in grasping or in their grip preferences. We 
also found that grey matter thickness within the CS fold was lower in chimpanzees than bonobos. Further, significantly greater leftward asymmetries were found in CS depth in chimpanzees compared to bonobos. In contrast, chimpanzees had greater rightward asymmetries in GM thickness compared to bonobos.

With respect to the hand preference results, the evidence that chimpanzees were significantly more right-handed than the bonobos, particularly for the TUBE task, suggest that previous reports of a lack of population-level handedness in bonobos are not an artefact of the sample size. From an evolutionary perspective, one interpretation of this finding is that selection for increasing motor skill, such as tool-use, may have resulted in increased preferential use of the right hand for coordinated bimanual actions in Pan troglodytes. The absence of selection for tool-use in bonobos may explain the less developed hand preferences in this species, at least with regard to the measures of handedness employed in this study. In short, the results of this study favour theories that emphasize the importance of tool-use as a potentially important adaptation for the development of right-handedness in early humans. There are, however, two caveats to this conclusion. First, the best way of directly testing for the potential role of the evolution of tool use on behavioural asymmetries would be to compare data from both bonobos and chimpanzees on tool use tasks. That was not done in this study but these types of data would lead to more definitive conclusions. Second, evidence of hand preference for tool use in wild chimpanzees is not entirely consistent with the theory that increased selection for tool use led to increased right-handedness. When data from wild chimpanzees are combined across field sites, they appear to show right-handedness for wadge dipping and ant -dipping but left hand preferences for termite fishing and no bias for nut-cracking. Of course, it may be the case that tool use per se was not the sole driving force behind the development of human right handedness. For example, though gorillas have been documented to use tools in captivity (Boysen et al., 1999; Parker et al., 1999), evidence of tool use in wild individuals, like bonobos, are fairly limited despite long-term behavioural observations at some field sites. However, of all the great apes and despite early claims by some (McGrew \& Marchant, 1993), gorillas appear to show the most consistent evidence of right handedness. For bimanual feeding, there is evidence of right-handedness in wild and captive populations (Byrne \& Byrne, 1991; Meguerditchian et al., 2010a; Lambert, 2012; Tablowo \& 


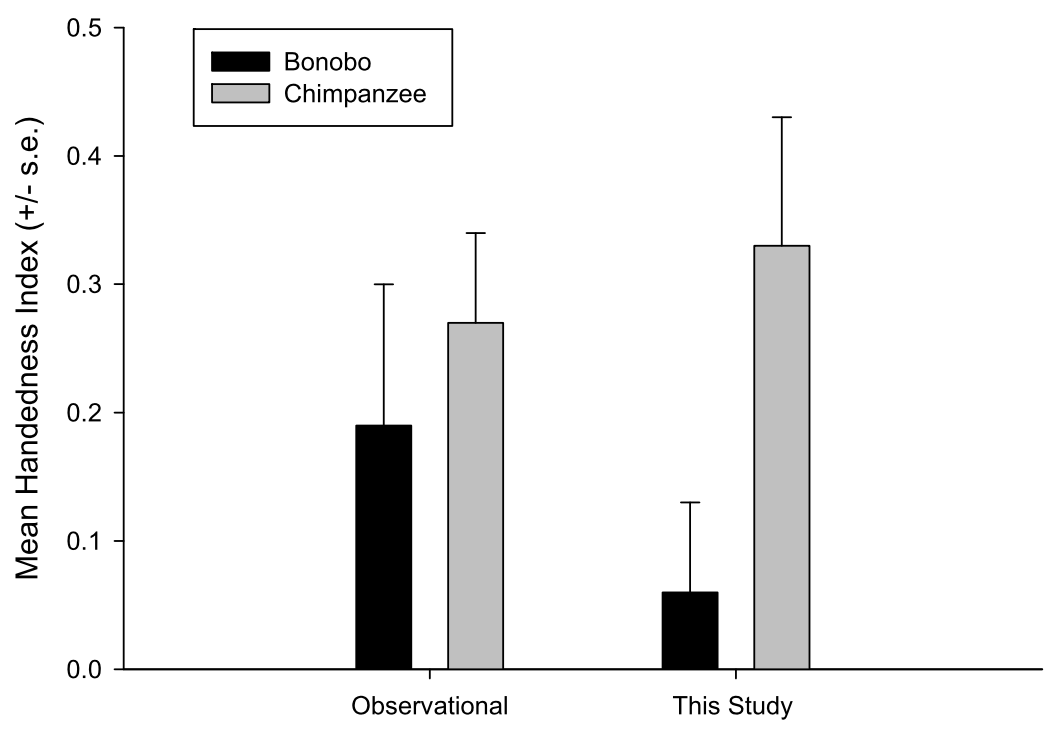

Figure 7. Mean HI scores $( \pm \mathrm{SE})$ in manual gestures from previous studies compared to the data reported in this study.

Forrester, 2013). Moreover, in two of three captive populations, more than $90 \%$ of the gorillas were reported to be right-handed for bimanual feeding. The fact that the chimpanzees were significantly more right-handed for all measures, including manual gestures, was somewhat surprising within the context of existing data on gestural communication in bonobos and chimpanzees. One factor that might explain this result is in the methodology used to assess handedness in chimpanzees and bonobos. Shown in Figure 7 are the mean HI scores for bonobos and chimpanzees for spontaneous communicative gestures pooled from published data in the literature (Hopkins \& de Waal, 1995; Shafer, 1997; Hopkins et al., 2005c, 2012; Harrison \& Nystrom, 2008; Meguerditchian et al., 2010b). As can be seen, the HI scores are significantly higher in the bonobos during the observational conditions compared to the results reported here, when collected under structured testing conditions. In contrast, the chimpanzee HI scores are relatively comparable between the observational and experimental conditions. Although we cannot rule out the possibility that the differences in $\mathrm{HI}$ values between the two bonobo cohorts simply reflects sampling variation, the results suggest that the experimental paradigm used in this study to elicit manual gestures did not induce a population-bias, which appears evident when assessing their 
natural interspecies communication. This discrepancy was less apparent in the chimpanzees and we would therefore caution against making too strong of a claim regarding the handedness for manual gestures in the bonobos.

The reason for the discrepancy in findings between the two bonobo cohorts is not clear but we would note that the tendency for the bonobos in this study to spontaneously point to foods outside of their home cage was rarely observed. This is quite different from what has been reported in captive living chimpanzees, particularly those housed at the Yerkes National Primate Research Center (Leavens \& Hopkins, 1998). In fact, at one zoo, a carestaff member indicated that the bonobos were discouraged from pointing or begging for foods from the humans interacting with them, thereby fundamentally extinguishing this behaviour. To what extent these types of experiences may influence both the propensity and lateralization in gestural communication during these interspecies exchanges is unclear; however, this could prove to be an important variable. Previous studies have shown that hand preferences for reaching at 4 years of age predicts subsequent hand preferences for manual gestures in captive chimpanzees as assessed by the methods employed in this study (Hopkins et al., 2005a). Hopkins et al. (2005a) have previously argued that these early reaching responses become ritualized into a manual gestural response as the subjects grow older. Early in life the young chimpanzees can reach through the cage mesh to retrieve food and they exhibit asymmetries in hand preference for this reaching response (Hopkins, 1995b). As they grow older, their hands become too large to reach through the cage mesh and therefore they develop a more ritualized gestural response to request the food. Thus, their hand preferences for manual gestures largely develop from their early reaching preferences. What may have happened with the bonobos in this study, compared to the chimpanzees, is that these ritualized processes during development were never experienced and therefore they did not come to use gestures functionally the same way as the chimpanzees, or at least those in this study. Though this is speculative, it is plausible and warrants further investigation.

Regarding grasping morphology, the results are largely consistent with the previous report by Christel et al. (1998) with bonobos showing a preponderance of thumb-index grasping responses. Though the findings reported by Christel et al. (1988) in bonobos were certainly suggestive of species differences when compared to previous reports on grasping morphology in chimpanzees (Tonooka \& Matsuzawa, 1995; Jones-Engel \& Bard, 1996; 
Butterworth \& Itakura, 1998; Hopkins et al., 2005d), our findings do not confirm the presence of differences in grasping morphology when subject variables such as age, sex and rearing history are controlled for in the species. We also found no significant differences in asymmetries in manual performance as measured by the error rates between the two species nor did we find any population-level biases within each species. Though the performance measures were not significant within and between species, the patterns of results between the hand preference and performance measures were consistent. That is to say, the chimpanzees showed population-level right hand preferences and show overall performance asymmetries which reflected better performance by the right compared to left hand on the grasping task. In contrast, the bonobos showed no population-level hand preferences and performed equally well with the right- and left-hands. The lack of significant population-level asymmetries in grasping performance differs from previous reports but this likely reflects the lower statistical power in this study compared to previous reports. Notably, Hopkins et al. (2002) assessed performance asymmetries in 74 chimpanzees in contrast to the 31 individuals in this group. Hopkins et al. (2002) noted that the effect size for performance asymmetries in grasping were relatively small and required larger samples in order to detect the differences. Thus, with only 31 chimpanzees and bonobos in this study, there may not have been enough statistical power to detect significant differences in grasping performance.

Analysis of the surface area, depth and GM thickness revealed several interesting results. Overall, the chimpanzees had thinner GM within the CS fold compared to bonobos. Previous studies have shown that GM thickness is negatively correlated with white matter volume in chimpanzees (Bogart et al., 2014); therefore, these results indicate that bonobos may have less white matter within the pre- or post-central gyri. Additionally, we found significant differences in asymmetries in mean depth and GM thickness with leftward asymmetries present in chimpanzees compared to bonobos for CS depth and the opposite pattern for GM thickness. These results are also consistent with the suggestion that differences in white matter between bonobos and chimpanzees in the pre- or post-central gyri may underlie the effects. It has been suggested that phylogenetic variation in cortical folding is driven, in part, by increases in white matter (van Essen, 1997; Herculano-Houzel et al., 2010). According to this view, regions that are strongly connected form more connections and results in the surface of the cortex folding inward and 
thereby forming gyri and sulci. By this definition, the greater CS depth in the left hemisphere of chimpanzees expands the surface area of the fold; the consequence is an increased thinning of the grey matter within the left hemisphere, which is necessary, assuming a finite and consistent amount of grey matter between hemispheres. Because the right hemisphere is less folded, it has grey matter that can be more thickly dispersed along the surface area.

Another important result was the differences in PPFM asymmetry. Though not significant at $p<0.05$, nonetheless, the data reported here are entirely consistent with the behavioural data on handedness (see Figure 6). Chimpanzees were more right-handed than the bonobos and they also showed much more rightward asymmetries in the PPFM. This may appear paradoxical but it is important to recall that a greater right AQ value for the PPFM actually reflects a larger region in the left hemisphere. Because we are measuring the depth of the fold, which is being impeded by the presence of the buried PPFM gyrus, a smaller PPFM value likely indicates a larger buried gyrus connecting the pre- and post-central gyri. Presumably this would indicate potentially greater intrahemispheric connectivity between the motor and somatosensory functions.

Finally, the robust leftward asymmetry in the PPFM found here in bonobos is consistent with the reported evidence of leftward asymmetries in the volume motor-hand region of the precentral gyrus or KNOB (Hopkins et al., 2009).

In summary, this study found that chimpanzees are significantly more right-handed than bonobos for a series of measures of hand preference. Like other studies on handedness in a different set of closely related species (Macaque, Cebus) (Spinozzi et al., 1998; Westergaard et al., 2001; Lilak \& Phillips, 2007; Meunier \& Vauclair, 2007), the results reported here suggest that differences in ecological or sociological factors may play an important role in the development of handedness in closely-related primates. There has historically been a tendency to consider primate handedness in very limited, linear evolutionary terms, with a very anthropocentric view of human uniqueness relative to other apes (Cashmore et al., 2008; Cashmore, 2009; Uomini, 2009). If nothing else, we believe our results suggest that many evolutionary factors influence the development of handedness and that there is need to more broadly consider the comparative study of handedness, particularly among closely-related species with the goal of trying to find common 
ecological or social factors that might explain between-species similarities and differences in handedness. This approach, we believe, may be more fruitful in identifying the unique factors that have selected for the pronounced expression of handedness seen in humans.

\section{Acknowledgements}

This research was supported in part by NIH grants NS-42867, NS-73134, HD-38051, and HD-56232 to WDH. O. Coulon is funded by the French National Research Agency (ANR-09-BLAN-0038-1, BrainMorph). The Yerkes Center is fully accredited by the American Association for Accreditation of Laboratory Animal Care. American Psychological Association guidelines for the ethical treatment of animals were adhered to during all aspects of this study. We are grateful to the helpful assistance of the bonobo veterinarians and keepers at the Milwaukee and Jacksonville Zoos, as well as the carestaff at the Iowa Primate Learning Center.

\section{References}

Alkadhi, H. \& Kollias, S.S. (2004). Pli de passage fronto-parietal moyen of Broca separates the motor homoculus. - Am. J. Neuroradiol. 25: 809-812.

Annett, M. (2002). Handedness and brain asymmetry: the right shift theory. — Psychology Press, Hove.

Bailey, P., von Bonin, G. \& McCulloch, W.S. (1950). The isocortex of the chimpanzee. University of Illinois Press, Urbana-Champaign, IL.

Biro, D., Inoue-Nakamura, N., Tonooka, R., Yamakoshi, G., Sousa, C. \& Matsuzawa, T. (2003). Cultural innovation and transmission of tool use in wild chimpanzees: evidence from field experiments. - Anim. Cogn. 6: 213-223.

Biro, D., Sousa, C. \& Matsuzawa, T. (2006). Ontogeny and cultural propagation of tool use by wild chimpanzees at Bossou, Guinea: case studies in nut cracking and leaf folding. In: Cognitive development of chimpanzees (Matsuzawa, T., Tomonaga, T. \& Tanaka, M., eds). Springer, New York, NY, p. 476-507.

Boesch, C. (1991). Handedness in wild chimpanzees. — Int. J. Primatol. 6: 541-558.

Bogart, S.L., Mangin, J.-F., Schapiro, S.J., Reamer, L., Bennett, A.J., Pierre, P.J. \& Hopkins, W.D. (2012a). Cortical sulci asymmetries in chimpanzees and macaques: a new look at an old idea. - NeuroImage 61: 533-541.

Bogart, S.L., Pruetz, J.D., Ormiston, L.K., Russell, J.L., Meguerditchian, A. \& Hopkins, W.D. (2012b). Termite fishing laterality in the Fongoli savanna chimpanzees (Pan troglodytes verus): further evidence of a left hand preference. — Am. J. Phys. Anthropol. 149: 591598. 
Bogart, S.L., Bennett, A.J., Schapiro, S.J., Reamer, L.A. \& Hopkins, W.D. (2014). Different early rearing experiences have long term effects on cortical organziation in captive chimpanzees (Pan troglodytes). — Dev. Sci. 17: 161-174.

Boling, W., Olivier, A., Bittar, R. \& Reutens, D. (1999). Localization of hand motor activation in Broca's pli de passage moyen. - J. Neurosurg. 91: 903-910.

Boysen, S.T., Kuhlmeier, K., Halliday, P. \& Halliday, Y.M. (1999). Tool use in captive gorillas. - In: The mentality of gorillas and orangutans (Parker, S.T., Mitchell, R.W. \& Miles, H.L., eds). Cambridge University Press, Cambridge.

Bradshaw, B. \& Rogers, L. (1993). The evolution of lateral asymmetries, language, tool-use and intellect. - Academic Press, San Diego, CA.

Bradshaw, J.L. (1997). Human evolution: a neuropsychological perspective. — Psychology Press, Hove.

Brakke, K.E. \& Savage-Rumbaugh, E.S. (1995). The development of language skills in bonobo and chimpanzee - I. Comprehension. - Lang. Commun. 15: 121-148.

Brakke, K.E. \& Savage-Rumbaugh, E.S. (1996). The development of language skills in Pan - II. Production. — Lang. Commun. 16: 361-380.

Butterworth, G. \& Itakura, S. (1998). Development of precision grips in chimpanzees. Dev. Sci. 1: 39-43.

Byrne, R.W. \& Byrne, J.M. (1991). Hand preferences in the skilled gathering tasks of mountain gorillas (Gorilla gorilla berengei). - Cortex 27: 521-536.

Cashmore, L. (2009). Can hominin "handedness" be accuartely assessed? — Ann. Hum. Biol. 36: 624-641.

Cashmore, L., Uomini, N. \& Chapelain, A. (2008). The evolution of handedness in humans and great apes: a review and current issues. - J. Anthropol. Sci. 86: 7-35.

Chapelain, A. \& Hogervorst, E. (2009). Hand preferences for bimanual coordination in 29 bonobos (Pan paniscus). — Behav. Brain Res. 196: 15-29.

Chapelain, A., Hogervorst, E., Mbonzo, P. \& Hopkins, W.D. (2011). Hand preferences for bimanual coordination in 77 bonobos (Pan paniscus): replication and extension. - Int. J. Primatol. 32: 491-510.

Christel, M.I., Kitzel, S. \& Niemitz, C. (1998). How precisely do bonobos (Pan paniscus) grasp small objects? — Int. J. Primatol. 19: 165-194.

Clay, Z. \& Zuberbuhler, K. (2011). Bonobos extract meaning from call sequences. - PLoS One 6: e18786.

Corballis, M.C. (1992). The lopsided brain: Evolution of the generative mind. - Oxford University Press, New York, NY.

Corballis, M.C. (2002). From hand to mouth: the origins of language. - Princeton University Press, Princeton, NJ.

Coulon, O., Clouchoux, C., Operato, G., Dauchot, K., Sirigu, A. \& Anton, J.-L. (2006). Cortical localization via surface parameterization: a sulcus-based approach. - NeuroImage 31: S46.

Cykowski, M.D., Coulon, O., Kochunov, P.V., Amunts, K., Lancaster, J.L., Laird, A.R., Glahn, C. \& Fox, P.T. (2008). The central sulcus: an observer-independent characterization of sulcal landmarks and depth asymmetry. — Cerebr. Cort. 18: 1999-2009. 
Dadda, M., Cantalupo, C. \& Hopkins, W.D. (2006). Further evidence of an association between handedness and neuroanatomical asymmetries in the primary cortex of chimpanzees (Pan troglodytes). — Neuropsychologia 44: 2482-2486.

Davatzikos, C. \& Bryan, R.N. (2002). Morphometric analysis of cortical sulci using parametric ribbons: a study of the central sulcus. — J. Comput. Assis. Tomogr. 26: 298-307.

de Waal, F.B.M. (1989). Behavioral contrasts between bonobo and chimpanzee. - In: Understanding chimpanzees (Heltne, P.G. \& Marquardt, L.A., eds). Harvard University Press, Cambridge, MA, p. 154-175.

Fischer, A., Wiebe, V., Pääbo, S. \& Przeworski, M. (2004). Evidence for a complex demographic history of chimpanzees. - Mol. Biol. Evol. 21: 799-808.

Forrester, G.S., Leavens, D.A., Quaresmini, C. \& Vallortigara, G. (2011). Target animacy influences gorilla handedness. - Anim. Cogn. 14: 903-907.

Forrester, G.S., Quaresmini, C., Leavens, D.A., Spiezio, C. \& Vallortigara, G. (2012). Target animacy influences chimpanzee handedness. - Anim. Cogn. 15: 1121-1127.

Frost, G.T. (1980). Tool behavior and the origin of laterality. — J. Hum. Evol. 9: 447-459.

Gallup, G.G. (1970). Chimpanzees: self-recognition. — Science 167: 86-87.

Gibson, K.R. \& Ingold, T. (1993). Tools, language and cognition in human evolution. Cambridge University Press, Cambridge.

Greenfield, P.M. (1991). Language, tools, and brain: the ontogeny and phylogeny of hierarchically organized sequential behavior. - Behav. Brain Sci. 14: 531-550.

Greenfield, P.M. \& Savage-Rumbaugh, E.S. (1990). Grammatical combination in Pan paniscus: processes of learning and invention in the evolution and development of language. In: "Language" and intelligence in monkeys and apes: comparative developmental perspectives (Parker, S.T. \& Gibson, K.R., eds). Cambridge University Press, New York, NY, p. 540-578.

Gruber, T., Clay, Z. \& Zuberbuhler, K. (2010). A comparison of bonobo and chimpanzee tool use: evidence for a femle bias in the Pan lineage. - Anim. Behav. 80: 1023-1033.

Hammond, G. (2002). Correlates of human handedness in primary motor cortex: a review and hypothesis. — Neurosci. Biobehav. Rev. 26: 285-292.

Hare, B. (2007). From nonhuman to human mind. what changed and why? - Curr. Direct. Psychol. Sci. 16: 60-64.

Hare, B. \& Tomasello, M. (2004). Chimpanzees are more skillful in competitive than in cooperative cognitive tasks. - Anim. Behav. 68: 571-581.

Hare, B., Melis, A.P., Woods, V., Hastings, S. \& Wrangham, R. (2007). Tolerance allows bonobos to outperform chimpanzees on a cooperative task. — Curr. Biol. 17: 619-623.

Harrison, R.M. \& Nystrom, P. (2008). Handedness in captive bonobos (Pan paniscus). Folia Primatologica 79: 253-268.

Herculano-Houzel, S., Mota, B., Wong, P. \& Kaas, J.H. (2010). Connectivity-driven white matter scaling and folding in primate cerebral cortex. — Proc. Natl. Acad. Sci. USA 107: 19008-19013.

Herrmann, E., Hare, B., Call, J. \& Tomasello, M. (2010). Differences in the cognitive skills of bonobos and chimpanzees. — PLoS One 5: e12438. 
Hopkins, W.D. (1995a). Hand preferences for a coordinated bimanual task in 110 chimpanzees: cross-sectional analysis. — J. Comp. Psychol. 109: 291-297.

Hopkins, W.D. (1995b). Hand preferences in juvenile chimpanzees: continuity in development. — Dev. Psychol. 31: 619-625.

Hopkins, W.D. (2007). Hemispheric specialization in chimpanzees: evolution of hand and brain. - In: Evolutionary cognitive neuroscience (Shackelford, T., Keenan, J.P. \& Platek, S.M., eds). MIT Press, Cambridge, MA, p. 99-120.

Hopkins, W.D. \& Cantalupo, C. (2004). Handedness in chimpanzees is associated with asymmetries in the primary motor but not with homologous language areas. - Behav. Neurosci. 118: 1176-1183.

Hopkins, W.D. \& de Waal, F.B.M. (1995). Behavioral laterality in captive bonobos (Pan paniscus): replication and extension. - Int. J. Primatol. 16: 261-276.

Hopkins, W.D. \& Pilcher, D.L. (2001). Neuroanatomical localization of the motor hand area with magnetic resonance imaging: the left hemisphere is larger in Great Apes. - Behav. Neurosci. 115: 1159-1164.

Hopkins, W.D. \& Russell, J.L. (2004). Further evidence of a right hand advantage in motor skill by chimpanzees (Pan troglodytes). — Neuropsychologia 42: 990-996.

Hopkins, W.D. \& Savage-Rumbaugh, E.S. (1991). Vocal communication as a function of differential rearing experiences in Pan paniscus: a preliminary report. — Int. J. Primatol. 12: 559-583.

Hopkins, W.D., Cantalupo, C., Wesley, M.J., Hostetter, A.B. \& Pilcher, D. (2002). Grip morphology and hand use in chimpanzees (Pan troglodytes): evidence of a left hemisphere specialization in motor skill. — J. Exp. Psychol. 131: 412-423.

Hopkins, W.D., Wesley, M.J., Izard, M.K., Hook, M. \& Schapiro, S.J. (2004). Chimpanzees are predominantly right-handed: replication in three colonies of apes. - Behav. Neurosci. 118: 659-663.

Hopkins, W.D., Russell, J., Freeman, H., Buehler, N., Reynolds, E. \& Schapiro, S. (2005a). The distribution and development of handedness for manual gestures in captive chimpanzees (Pan troglodytes). — Psych. Sci. 16: 487.

Hopkins, W.D., Russell, J., Hook, M., Braccini, S. \& Schapiro, S. (2005b). Simple reaching is not so simple: association between hand use and grip preferences in captive chimpanzees. — Int. J. Primatol. 26: 259-277.

Hopkins, W.D., Russell, J.L., Freeman, H., Buehler, N., Reynolds, E. \& Schapiro, S.J. (2005c). The distribution and development of handedness for manual gestures in captive chimpanzees (Pan troglodytes). — Psychol. Sci. 16: 487-493.

Hopkins, W.D., Russell, J.L., Hook, M., Braccini, S. \& Schapiro, S.J. (2005d). Simple reaching is not so simple: association between hand use and grip preferences in captive chimpanzees. — Int. J. Primatol. 26: 259-277.

Hopkins, W.D., Russell, J.L. \& Cantalupo, C. (2007). Neuroanatomical correlates of handedness for tool use in chimpanzees (Pan troglodytes): implication for theories on the evolution of language. - Psychol. Sci. 18: 971-977. 
Hopkins, W.D., Lyn, H. \& Cantalupo, C. (2009). Volumetric and lateralized differences in selected brain regions of chimpanzees (Pan troglodytes) and bonobos (Pan paniscus). Am. J. Primatol. 71: 988-997.

Hopkins, W.D., Coulon, O. \& Mangin, J.F. (2010). Observer-independent characterization of sulcal landmarks and depth asymmetry in the central sulcus of the chimpanzee brain. Neuroscience 171: 544-551.

Hopkins, W.D., Phillips, K.A., Bania, A., Calcutt, S.E., Gardner, M., Russell, J.L., Schaeffer, J.A., von Lonsdorf, E., Ross, S. \& Schapiro, S.J. (2011). Hand preferences for coordinated bimanual actions in 777 great apes: implications for the evolution of handedness in Hominins. - J. Hum. Evol. 60: 605-611.

Hopkins, W.D., Pika, S., Liebal, K., Bania, A., Meguerditchian, A., Gardner, M. \& Schapiro, S.J. (2012). Handedness for manual gestures in great apes: a meta-analysis. — In: Current developments in non-human primate gesture research (Pika, S. \& Liebal, K., eds). John Benjamins Publishing, Amsterdam.

Humle, T. \& Matsuzawa, T. (2009). Laterality in hand use across four tool use behaviors among the wild chimpanzees of Bossou, Guinea, West Africa. - Am. J. Primatol. 71: 40-48.

Hyatt, C.W. \& Hopkins, W.D. (1994). Self-awareness in bonobos and chimpanzees: a comparative perspective. - In: Self-awareness in animals and humans: developmental perspectives (Parker, S.T., Mitchell, R.W. \& Boccia, M.L., eds). Cambridge University Press, New York, NY, p. 248-253.

Jones-Engel, L.E. \& Bard, K.A. (1996). Precision grips in young chimpanzees. - Am. J. Primatol. 39: 1-15.

Lambert, M. (2012). Hand preference for bimanual and unimanual feeding in captive gorillas: extension in a second colony of apes. - Am. J. Phys. Anthropol. 148: 641-647.

Leavens, D.A. \& Hopkins, W.D. (1998). Intentional communication by chimpanzee (Pan troglodytes): a cross-sectional study of the use of referential gestures. - Dev. Psychol. 34: 813-822.

Lilak, A.L. \& Phillips, K.A. (2007). Consistency in hand preference across low-level and high-level tasks in capuchin monkeys (Cebus apella). - Am. J. Primatol. 69: 1-12.

Llorente, M., Palou, L., Carrasco, L., Riba, D., Mosquera, M., Colell, M., Fabre, M. \& Feliu, O. (2010). Population-level right handedness for a coordinated bimanual task in naturalistic housed chimpanzees: replication and extension in 114 animals from Zambia and Spain. - Am. J. Primatol. 71: 1-10.

Lyn, H. (2007). Mental representation of symbols as revealed by vocabulary errors in two bonobos (Pan paniscus). - Anim. Cogn. 10: 461-475.

Lyn, H., Greenfield, P.M. \& Savage-Rumbaugh, E.S. (2006). The development of representational play in chimpanzees and bonobos: evolutionary implications, pretense, and the role of interspecies communication. - Cogn. Dev. 21: 199-213.

Lyn, H., Greenfield, P.M. \& Savage-Rumbaugh, E.S. (2011). Semiotic combinations in Pan: a cross-species comparison of communication in a chimpanzee and a bonobo. - First Lang. 31: 300-325. 
Mangin, J.F., Riviere, D., Cachia, A., Duchesnay, E., Cointepas, Y., Papadopoulos-Orfanos, D., Collins, D.L., Evans, A.C. \& Regis, J. (2004). Object-based morphometry of the cerebral cortex. - Med. Imag. 23: 968-982.

Marchant, L.F. \& McGrew, W.C. (2007). Ant fishing by wild chimpanzees is not lateralised. — Primates 48: 22-26.

McGrew, W.C. (1992). Chimpanzee material culture: implications for human evolution. Cambridge University Press, Cambridge.

McGrew, W.C. \& Marchant, L.F. (1992). Chimpanzees, tools, and termites: hand preference or handedness? - Curr. Anthropol. 33: 114-119.

McGrew, W.C. \& Marchant, L.F. (1993). Are gorillas right-handed or not? - Hum. Evol. 8: $17-23$

McGrew, W.C. \& Marchant, L.F. (1996). On which side of the apes? - In: Great ape societies (McGrew, W.C., Marchant, L.F. \& Nishida, T., eds). Cambridge University Press, Cambridge, p. 255-272.

McGrew, W.C. \& Marchant, L.F. (1997). Using the tools at hand: manual laterality and elementary technology in Cebus spp. and Pan spp. — Int. J. Primatol. 18: 787-810.

Meguerditchian, A., Calcutt, S.E., von Lonsdorf, E., Ross, S.R. \& Hopkins, W.D. (2010a). Captive gorillas are right-handed for bimanual feeding. - Am. J. Phys. Anthropol. 141: 638-645.

Meguerditchian, A., Vauclair, J. \& Hopkins, W.D. (2010b). Captive chimpanzees use their right hand to communicate with each other: implications for the origins of hemispheric specialization for language. - Cortex 46: 40-48.

Meunier, H. \& Vauclair, J. (2007). Hand preferences on unimanual and bimanual tasks in white-face capuchins. — Am. J. Primatol. 69: 1064-1069.

Parish, A.R. (1996). Female relationships in bonobos (Pan paniscus) — evidence for bonding, cooperation, and female dominance in a male-philopatric species. - Hum. Nat. Interdisc. Biosoc. Perspect. 7: 61-96.

Parish, A.R., de Waal, F.B.M. \& Haig, D. (2000). The other "closest living relative": how bonobos (Pan paniscus) challenge traditional assumptions about females, dominance, intra- and intersexual interactions, and hominid evolution. - Ann. NY Acad. Sci. 907: 97-113.

Parker, S., Kerr, M., Markowitz, H. \& Gould, J. (1999). A survey of tool use in zoo gorillas. — In: The mentalities of gorillas and orangutans: comparative perspectives (Parker, S.T. \& Mitchell, R.W., eds). Cambridge University Press, Cambridge.

Phillips, K.A. \& Sherwood, C.C. (2005). Primary motor cortex asymmetry is correlated with handedness in capuchin monkeys (Cebus apella). — Behav. Neurosci. 119: 1701-1704.

Pika, S., Liebal, K. \& Tomasello, M. (2005). The gestural repertoire of bonobos (Pan paniscus): flexibility and use. - Am. J. Primatol. 65: 39-61.

Pollick, A.S. \& de Waal, F.B.M. (2007). Ape gestures and language evolution. — Proc. Natl. Acad. Sci. USA 104: 8184-8189.

Rilling, J.K., Scholz, J., Preuss, T.M., Glasser, M.F., Errangi, B.V. \& Behrens, T.E.J. (2012). Differences between chimpanzees and bonobos in neural systems supporting social cognition. - Soc. Cogn. Affect. Neurosci. 7: 369-379. 
Rosati, A.G. \& Hare, B. (2012). Chimpanzees and bonobos exhibit divergent spatial memory development. - Dev. Sci. 15: 840-853.

Savage-Rumbaugh, E.S. (1984). Pan paniscus and Pan troglodytes: contrast in preverbal communicative competence. - In: The pygmy chimpanzee: evolutionary biology and behavior (Susman, R.L., ed.). Plenum Press, New York, NY, p. 395-413.

Shafer, D.D. (1997). Hand preference behaviors shared by two groups of captive bonobos. Primates 38: 303-313.

Spinozzi, G., Castornina, M.G. \& Truppa, V. (1998). Hand preferences for unimanual and coordinated-bimanual tasks in tufted capuchin monkeys (Cebus apella). - J. Comp. Psychol. 112: 183-191.

Stanford, C.B. (1998). The social behavior of chimpanzees and bonobos: empirical evidence and shifting assumptions. - Curr. Anthropol. 39: 399-420.

Tablowo, E. \& Forrester, G.S. (2013). Stuctured bimanual actions and hand transfers reveal population-level right handedness in captive gorillas. - Anim. Behav. 86: 1049-1057.

Taglialatela, J.P., Savage-Rumbaugh, E.S. \& Baker, L.A. (2003). Vocal production by a language-competent Pan paniscus. - Int. J. Primatol. 24: 1-17.

Tonooka, R. \& Matsuzawa, T. (1995). Hand preferences in captive chimpanzees (Pan troglodytes) in simple reaching for food. - Int. J. Primatol. 16: 17-34.

Toth, N., Schick, K.D., Savage-Rumbaugh, E.S., Sevcik, R.A. \& Rumbaugh, D.M. (1993). Pan the tool-maker: investigations into the stone tool-making and tool-using capabilities of a bonobo (Pan paniscus). - J. Archaeol. Sci. 20: 81-91.

Uomini, N.T. (2009). The prehistory of handedness: archeological data and comparative ethology. - J. Hum. Evol. 57: 411-419.

van Essen, D.C. (1997). A tension-based theory of morphogenesis and compact wiring in the central nervous system. - Nature 385: 313-318.

von Lonsdorf, E. \& Hopkins, W.D. (2005). Wild chimpanzees show population level handedness for tool use. - Proc. Natl. Acad. Sci. USA 102: 12634-12638.

Westergaard, G.C., Lussier, I.D. \& Higley, J.D. (2001). Between-species variation in the development of hand preference among macaques. - Neuropsychologia 39: 1373-1378.

Whiten, A., Goodall, J., McGrew, W., Nishida, T., Reynolds, V., Sugiyama, Y., Tutin, C., Wrangham, R. \& Boesch, C. (2001). Charting cultural variation in chimpanzees. - Behaviour 138: 1489-1525.

Whiten, A., Goodall, J., McGrew, W.C., Nishida, T., Reynolds, V., Sugiyama, Y., Tutin, C.E.G., Wrangham, R.W. \& Boesch, C. (1999). Cultures in chimpanzees. - Nature 399: 682-685.

Won, Y.-J. \& Hey, J. (2005). Divergence population genetics of chimpanzees. - Mol. Biol. Evol. 22: 297-307.

Yousry, T.A., Schmid, U.D., Alkadhi, H., Schmidt, D., Peraud, A., Buettner, A. \& Winkler, P. (1997). Localization of the motor hand area to a knob on the precentral gyrus. A new landmark. — Brain 120: 141-157. 